\title{
Protective effects of acute exercise preconditioning on disuse-induced muscular atrophy in aged muscle: a narrative literature review
}

\author{
Toshinori Yoshihara* ${ }^{*}$ and Hisashi Naito
}

\begin{abstract}
Aging is associated with a progressive loss of skeletal muscle mass and strength, resulting in frailty and lower quality of life in older individuals. At present, a standard of clinical or pharmacological care to prevent the adverse effects of aging does not exist. Determining the mechanism(s) responsible for muscular atrophy in disused aged muscle is a required key step for the development of effective countermeasures. Studies suggest an age-related differential response of genes and signalings to muscle disuse in both rodents and humans, implying the possibility that effective countermeasures to prevent disuse muscle atrophy may be age-specific. Notably, exercise preconditioning can attenuate disuse-induced muscular atrophy in rodent and human skeletal muscles; however, information on age-specific mechanisms of this exercise-induced protection remains limited. This mini-review aimed to summarize the protective effects of acute exercise preconditioning on muscular atrophy in aged muscle and provide potential mechanisms for its preventive effect on skeletal muscle wasting.
\end{abstract}

Keywords: Growth arrest and DNA damage-inducible 45a, Histone deacetylase 4, Exercise intervention, Aged skeletal muscle, Sarcopenia

\section{Background}

Aging is associated with frailty, impaired health span, and lower quality of life [1]. Aging impacts muscle adaptations, such as muscle hypertrophy, increased antioxidant capacity, muscle regeneration, and muscle atrophy. Interestingly, several studies have demonstrated that there are age-specific gene and signaling responses to skeletal muscle disuse in both rodents and humans [2-6]. For instance, Leeuwenburgh et al. demonstrated that old rats (32 months old) have a greater apoptotic response to hindlimb unloading in rat soleus muscle than in young rats (6 months old), suggesting that apoptotic

*Correspondence: t-yoshih@juntendo.ac.jp

Graduate School of Health and Sports Science, Juntendo University, 1-1

Hirakagakuendai, Inzai, Chiba 270-1695, Japan regulation during disuse is distinct in young and aged muscles [2]. In human skeletal muscle, one paper comparing age-related differential mechanisms in disuse muscle atrophy (21-27 years vs. 60-72 years) found an age-specific upregulation of Bax and p53 in aged muscle after 2 days of immobility with significant increases in TdT-mediated dUTP nick end labeling and DNA fragmentation in old muscle [6]. Moreover, recent evidence has revealed that growth arrest and DNA damageinducible $45 \alpha$ (Gadd $45 \alpha)$ are required for skeletal muscle atrophy induced by different muscle stressors, such as fasting, denervation, and immobilization [7]. Gadd $45 \alpha$ is a soluble, primarily myonuclear protein that causes muscle fiber atrophy by altering skeletal muscle gene expression, stimulating protein breakdown, reducing protein synthesis, decreasing mitochondria, and inhibiting anabolic signaling [7]. It is notable that Gadd $45 \alpha$

(c) The Author(s) 2020. This article is licensed under a Creative Commons Attribution 4.0 International License, which permits use, sharing, adaptation, distribution and reproduction in any medium or format, as long as you give appropriate credit to the original author(s) and the source, provide a link to the Creative Commons licence, and indicate if changes were made. The images or other third party material in this article are included in the article's Creative Commons licence, unless indicated otherwise in a credit line to the material. If material is not included in the article's Creative Commons licence and your intended use is not permitted by statutory regulation or exceeds the permitted use, you will need to obtain permission directly from the copyright holder. To view a copy of this licence, visit http://creativeco mmons.org/licenses/by/4.0/. 
mRNA expression is significantly increased in the tibialis anterior muscle in 29-month-old rats after 14 days of hindlimb unloading, but not in 9-month-old rats [8]. This suggests that Gadd $45 \alpha$ plays a key role in disuse-induced muscle atrophy, especially in aged skeletal muscle. To preserve the functionality of aged muscle, it is important to develop therapeutic countermeasures prior to muscle disuse and identify target molecules that protect against disuse-induced skeletal muscle atrophy.

Although various countermeasures have been developed as potential therapeutic treatments to protect against disuse skeletal muscle atrophy, endurance exercise preconditioning is one of the most practical countermeasures currently available. Even a single bout of preconditioning exercise can attenuate disuse muscle atrophy induced by hindlimb unloading in rats [9]. This evidence suggests that a single bout of preconditioning exercise may be a simple and effective countermeasure against disuse muscle atrophy in aged skeletal muscle. While most older people's circumstances or physical conditions do not permit exercise prior to the atrophic situation, investigating the mechanism(s) responsible for preconditioning exercise-induced protection against disuse muscle atrophy provides unique information to identify biological targets for intervention. By identifying these biological targets, we can develop future therapeutic approaches to prevent muscle wasting. However, at present, there is limited supporting evidence for exercise preconditioning-induced protection against disuseinduced muscle atrophy in aged muscle.

\section{Differential signaling responses to disuse in aged muscle}

Aging affects gene and signaling responses to skeletal muscle disuse in rodents and humans, and investigators have demonstrated that some apoptotic responses to disuse are age-specific [2-6]. Interestingly, recent evidence indicated that growth arrest and DNA damage-inducible $45 \alpha$ (Gadd $45 \alpha)$ is required for skeletal muscle atrophy induced by different muscle stressors, such as fasting, denervation, and immobilization [7]. Gadd $45 \alpha$ is a soluble, primarily myonuclear protein that alters skeletal muscle gene expression and stimulates protein breakdown, reduces protein synthesis, decreases mitochondria, activates apoptosis, and consequently causes muscle fiber atrophy [7]. Furthermore, histone deacetylase (HDAC) 4 , a class II histone deacetylase, is an important regulator of Gadd $45 \alpha$ in denervation-induced muscle atrophy [10]. Interestingly, the previous study indicated that Gadd $45 \alpha$ mRNA expression was significantly increased in the tibialis anterior muscle in old rats (29 months old) after 14 days of hindlimb unloading, but not in young adult rats (9 months old) [8]. Moreover, Baehr et al. demonstrated that Gadd $45 \alpha$ mRNA expression increased significantly after 3 and 7 days of hindlimb unloading in the gastrocnemius muscle and that old rats (29 months old) showed greater Gadd $45 \alpha$ mRNA expression for the entire unloading period compared with young adult rats (9 months old) [5]. Activation of these pathways induced the age-related delay in recovery from atrophy [8]. These facts suggest that HDAC4/Gadd $45 \alpha$ axis plays an important role in hindlimb unloading-induced muscle atrophy in aged skeletal muscle; thus, HDAC4/Gadd $45 \alpha$ axis may be a key pathway for developing potential therapeutic countermeasures in aged muscles before muscle disuse and in identifying target molecules to protect against disuse skeletal muscle atrophy.

\section{Protective effect of exercise on disuse muscle atrophy in aged muscle}

Until now, a variety of countermeasures have been investigated as potential treatments to protect against disuse skeletal muscle atrophy in humans. Physical strategies, such as resistance exercises and maximal voluntary contractions, which can be performed both isometrically and dynamically, are feasible during most immobilization situations and represent powerful tools for preventing muscle atrophy [11]. Moreover, regular exercise can attenuate the major hallmarks of aging, such as genomic instability, loss of proteostasis, mitochondrial dysfunction, cellular senescence, and age-related muscle wasting $[12,13]$. Based on previous human studies, exercise, particularly resistance exercise, is a practical countermeasure to age-related muscle atrophy; however, the underlying mechanisms by which exercise preconditioning may prevent adverse effects on aging muscles remain unknown. Notably, even a single bout of preconditioning exercise can attenuate disuse muscle atrophy induced by hindlimb unloading in the rat. For instance, Fujino et al. [9] demonstrated that a bout of exercise preconditioning $\left(20^{\circ}\right.$ slope, $\left.20 \mathrm{~m} / \mathrm{min}, 25 \mathrm{~min}\right)$ without pre-familiarization before 2 weeks of hindlimb unloading attenuated slow-type soleus muscle atrophy by preventing mRNA expressions of the proteolytic pathway (cathepsin L, calpain, caspase-3, and E3 ubiquitin ligases) in 9- to 10-week-old male Wistar rats. This suggests that a single bout of preconditioning exercise may effectively suppress the upregulation of protein degradation during disuse in aged skeletal muscle. Additionally, we recently demonstrated that a bout of exercise could suppress Gadd $45 \alpha$ upregulation in the gastrocnemius muscle of old rats [14]. As Gadd45 $\alpha$ appears to have an age-specific change in response to hindlimb unloading, it is a potential target for exercise-induced protection against disuse muscle atrophy. Moreover, recent work has demonstrated that HDAC4 is an important regulator of 
Gadd $45 \alpha$ in denervation-induced muscle atrophy, and the knockout of HDAC4 can attenuate denervationinduced muscle atrophy [10]. Therefore, exercise preconditioning-induced prevention of Gadd $45 \alpha$ via HDAC4 should be effective for aged muscle atrophy. Similarly, we found that acute exercise preconditioning $\left(0^{\circ}\right.$ slope, $20 \mathrm{~m} / \mathrm{min}, 15 \mathrm{~min}$ ) without pre-familiarization can prevent HDAC4 protein and mRNA upregulation in old rats (24-month-old male Wistar rats) with the prevention of downstream Gadd $45 \alpha$ [14]. This study's exercise protocol was relatively low-intensity and short-duration compared with the previous study performed by Fujino et al.; therefore, no protective effect was observed in 3-month-old (young) rats.
Although the precise mechanisms of acute exercise preconditioning-induced protection against muscle atrophy are still unknown, there are some candidate mechanisms that indicate exercise can prevent HDAC4/Gadd45 $\alpha$ pathway upregulation in aged muscle (Fig. 1). First, a previous study reported that Gadd $45 \alpha$ reduces multiple barriers to muscle atrophy, including peroxisome proliferator-activated receptor-gamma coactivator-1 (PGC-1 $\alpha)$ [7]. PGC-1 $\alpha$ plays a crucial role in the exercise-induced regulation of muscle atrophy, mitochondrial biogenesis, energy metabolism, and muscle fiber type in old mice skeletal muscle [15]; thus, preserving PGC-1 $\alpha$ expression via Gadd $45 \alpha$ downregulation seems to be one of the key factors of exercise-induced protection against

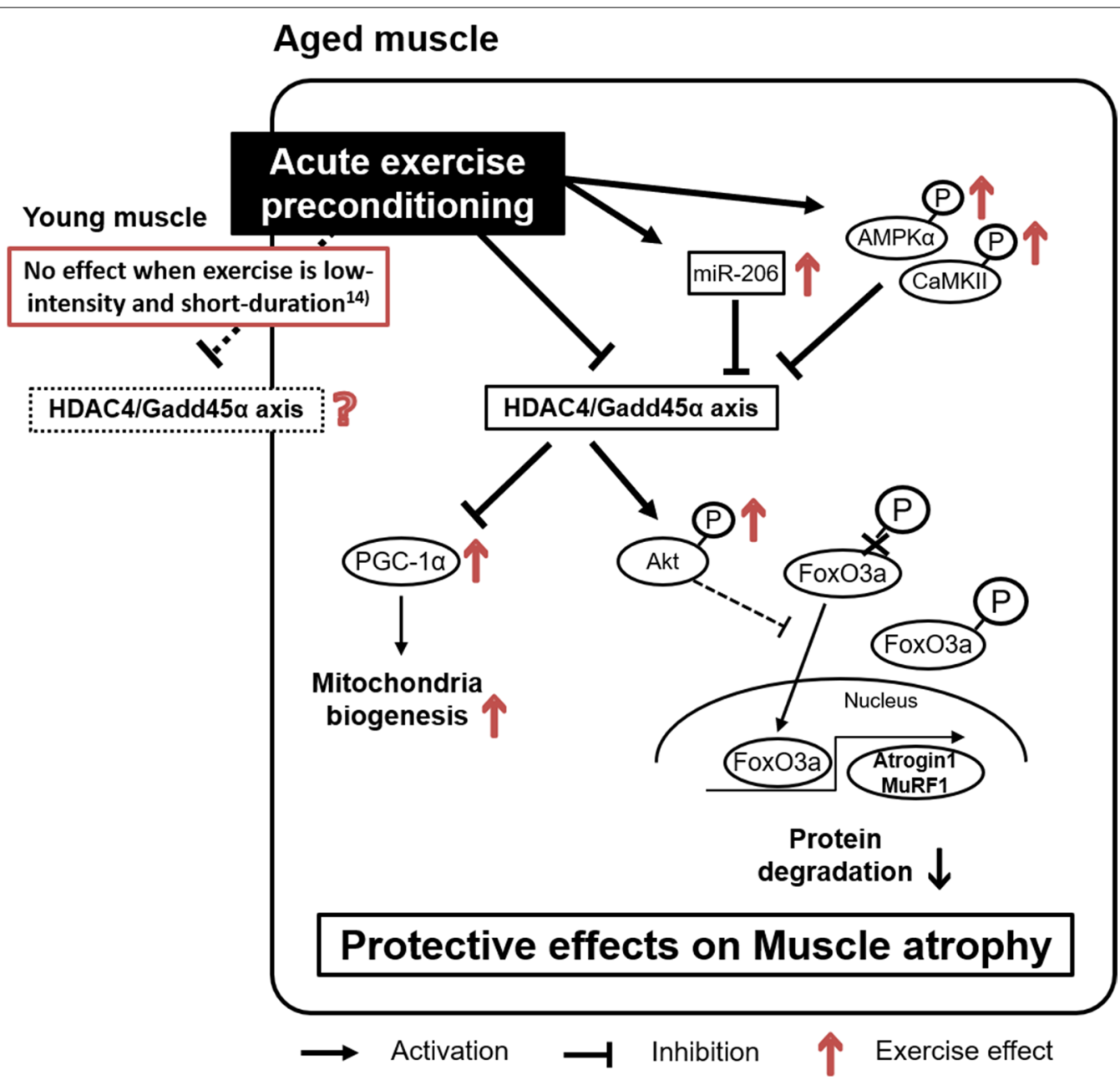

Fig. 1 Potential underlying mechanisms of the protective effects of acute exercise preconditioning on disuse-induced muscular atrophy in aged muscle. HDAC4/Gadd45a axis plays an important role in disuse-induced muscle atrophy in aged skeletal muscle via preserving peroxisome proliferator-activated receptor-gamma coactivator-1 (PGC-1a) expression and maintaining Akt phosphorylation during disuse. Other potential mechanisms of exercise-induced protection against the HDAC4/Gadd45a axis include the upregulation of microRNA (miR)-206 and the phosphorylation of AMP-activated protein kinase a (AMPKa) and calcium/calmodulin-dependent kinase II (CaMKII). Arrows represent the exercise effects based on the results from the previous study [14] 
muscle atrophy. Second, the exercise preconditioninginduced protection against HDAC4/Gadd $45 \alpha$ pathway can maintain Akt phosphorylation during disuse [14]. Akt phosphorylation regulates FoxO3a phosphorylation $[16,17]$, leading to the exclusion of phosphorylated FoxO3a proteins from the nucleus and the inhibition of transcriptional function to induce the downregulation of E3 ligases. Thus, at least in part, it plays an important role in the protective effect on disuse muscle atrophy in old rats [14]. Additionally, microRNA (miR)-206, a member of muscle-enriched miRNAs, is known to facilitate muscle differentiation by regulating the expression of myogenic regulatory factors [18]. Previous studies have reported that miR-206 regulates HDAC4 expression in the muscle under atrophic conditions [18, 19]. Overexpression of miR-206 decreased endogenous HDAC4 levels in the tibialis anterior muscles of mice [20], and miR-206 can attenuate denervation-induced rat skeletal muscle atrophy through the HDAC4-related signaling [21]. In human skeletal muscle, $2 \mathrm{~h}$ of acute resistance exercise increased miR-206 [22], and 90 min of ergometer exercise increased miR-206, but load carriage treadmill running did not increase miR-206 [23], suggesting that the effect of acute exercise on miR-206 expression in humans might depend on the exercise conditions. Other potential mechanisms of exercise-induced protection against the HDAC4/Gadd $45 \alpha$ axis are the phosphorylation of AMP-activated protein kinase $\alpha$ (AMPK $\alpha)$ and calcium/calmodulin-dependent kinase II (CaMKII) [24]. In disuse conditions, such as limb immobilization, AMPK $\alpha$ and CaMKII are both de-activated and affect HDAC4 phosphorylation during muscle atrophy $[25,26]$, resulting in upregulation of Gadd $45 \alpha$ and E3 ligases. In contrast, exercise preconditioning reverses these effects and prevents HDAC4/Gadd45 $\alpha$ pathway upregulation in aged muscle. Nonetheless, the information on exerciseinduced protection against disuse muscle atrophy is still limited; therefore, future studies are required to clarify the mechanisms through which exercise prevents muscle atrophy in aged skeletal muscle.

\section{Conclusions}

The evidence suggests that acute exercise preconditioning is an effective countermeasure against a reduction in muscle mass in aged skeletal muscle, and the mechanisms of exercise-induced protection against skeletal muscle loss may be age-specific. Moreover, this protective effect in the aged muscle may be partially mediated by the HDAC4/Gadd $45 \alpha$ axis and subsequent protein degradation systems. Currently, supporting evidence in this area is limited, especially regarding the age-specific effects of chronic exercise on disuse-induced muscle atrophy. Therefore, future studies should clarify mechanisms of exercise-induced protection on muscular atrophy in aged muscle and lead to developing therapeutic approaches to prevent muscle wasting.

\section{Abbreviations \\ Gadd45a: Growth arrest and DNA damage-inducible 45a; HDAC: Histone deacetylase; PGC-1a: Peroxisome proliferator-activated receptor gamma coactivator-1a; miR: MicroRNA; AMPKa: AMP-activated protein kinase a; CaM- KII: Calcium/calmodulin-dependent kinase II.}

\section{Acknowledgements}

We would like to thank Editage (http://www.editage.com) for English language editing.

\section{Author's contributions}

TY drafted the manuscript; TY and HN edited and revised the manuscript; TY and $\mathrm{HN}$ approved the final version of the manuscript. All authors read and approved the final manuscript.

\section{Funding}

This work was supported by the Japan Society for the Promotion of Science KAKENHI (Grant No. 20H04082 and $17 \mathrm{~K} 01765$ to T. Yoshihara), and Ministry of Education, Culture, Sports, Science, and Technology Supported Program for the Strategic Research Foundation at Private Universities. This work was also supported in part by a collaborative research grant from the Juntendo University.

\section{Availability of data and materials}

Not applicable.

Ethics approval and consent to participate

Not applicable.

\section{Consent for publication}

Not applicable.

\section{Competing interests}

The authors have no personal, financial or nonfinancial conflict of interest.

Received: 12 June 2020 Accepted: 19 November 2020

Published online: 27 November 2020

\section{References}

1. Demontis F, Piccirillo R, Goldberg AL, Perrimon N (2013) Mechanisms of skeletal muscle aging: insights from Drosophila and mammalian models. Dis Model Mech 6(6):1339-1352

2. Leeuwenburgh C, Gurley CM, Strotman BA, Dupont-Versteegden EE (2005) Age-related differences in apoptosis with disuse atrophy in soleus muscle. Am J Physiol Regul Integr Comp Physiol 288(5):R1288-1296

3. Su J, Ekman C, Oskolkov N, Lahti L, Strom K, Brazma A, Groop L, Rung J, Hansson O (2015) A novel atlas of gene expression in human skeletal muscle reveals molecular changes associated with aging. Skelet Muscle 5:35

4. Marzetti E, Wohlgemuth SE, Lees HA, Chung HY, Giovannini S, Leeuwenburgh C (2008) Age-related activation of mitochondrial caspase-independent apoptotic signaling in rat gastrocnemius muscle. Mech Ageing Dev 129(9):542-549

5. Baehr LM, West DWD, Marshall AG, Marcotte GR, Baar K, Bodine SC (2017) Muscle-specific and age-related changes in protein synthesis and protein degradation in response to hindlimb unloading in rats. J Appl Physiol (1985) 122(5):1336-1350

6. Suetta C, Frandsen U, Jensen L, Jensen MM, Jespersen JG, Hvid LG, Bayer M, Petersson SJ, Schroder HD, Andersen JL et al (2012) Aging affects the transcriptional regulation of human skeletal muscle disuse atrophy. PLoS ONE 7(12):e51238 
7. Ebert SM, Dyle MC, Kunkel SD, Bullard SA, Bongers KS, Fox DK, Dierdorff JM, Foster ED, Adams CM (2012) Stress-induced skeletal muscle Gadd45a expression reprograms myonuclei and causes muscle atrophy. J Biol Chem 287(33):27290-27301

8. Baehr LM, West DW, Marcotte G, Marshall AG, De Sousa LG, Baar K, Bodine SC (2016) Age-related deficits in skeletal muscle recovery following disuse are associated with neuromuscular junction instability and ER stress, not impaired protein synthesis. Aging (Albany NY) 8(1):127-146

9. Fujino H, Ishihara A, Murakami S, Yasuhara T, Kondo H, Mohri S, Takeda I, Roy RR (2009) Protective effects of exercise preconditioning on hindlimb unloading-induced atrophy of rat soleus muscle. Acta Physiol (Oxf) 197(1):65-74

10. Bongers KS, Fox DK, Ebert SM, Kunkel SD, Dyle MC, Bullard SA, Dierdorff JM, Adams CM (2013) Skeletal muscle denervation causes skeletal muscle atrophy through a pathway that involves both Gadd45a and HDAC4. Am J Physiol Endocrinol Metab 305(7):E907-915

11. Valenzuela PL, Morales JS, Pareja-Galeano H, Izquierdo M, Emanuele E, de la Villa P, Lucia A (2018) Physical strategies to prevent disuse-induced functional decline in the elderly. Ageing Res Rev 47:80-88

12. Garatachea N, Pareja-Galeano H, Sanchis-Gomar F, Santos-Lozano A, Fiuza-Luces C, Moran M, Emanuele E, Joyner MJ, Lucia A (2015) Exercise attenuates the major hallmarks of aging. Rejuvenation Res 18(1):57-89

13. Yoo SZ, No MH, Heo JW, Park DH, Kang JH, Kim SH, Kwak HB (2018) Role of exercise in age-related sarcopenia. J Exerc Rehabil 14(4):551-558

14. Yoshihara T, Tsuzuki T, Chang SW, Kakigi R, Sugiura T, Naito H (2019) Exercise preconditioning attenuates hind limb unloading-induced gastrocnemius muscle atrophy possibly via the HDAC4/Gadd45 axis in old rats. Exp Gerontol 122:34-41

15. Theilen NT, Jeremic N, Weber GJ (1985) Tyagi SC (2018) Exercise preconditioning diminishes skeletal muscle atrophy after hindlimb suspension in mice. J Appl Physiol 125(4):999-1010

16. Yang W, Dolloff NG, El-Deiry WS (2008) ERK and MDM2 prey on FOXO3a. Nat Cell Biol 10(2):125-126

17. Brunet A, Bonni A, Zigmond MJ, Lin MZ, Juo P, Hu LS, Anderson MJ, Arden KC, Blenis J, Greenberg ME (1999) Akt promotes cell survival by phosphorylating and inhibiting a Forkhead transcription factor. Cell 96(6):857-868

18. Williams AH, Valdez G, Moresi V, Qi X, McAnally J, Elliott JL, Bassel-Duby R, Sanes JR, Olson EN (2009) MicroRNA-206 delays ALS progression and promotes regeneration of neuromuscular synapses in mice. Science 326(5959):1549-1554

19. Choi W, Lee J, Ko KR, Kim S (2018) Hepatocyte growth factor regulates the miR-206-HDAC4 cascade to control neurogenic muscle atrophy following surgical denervation in mice. Mol Ther Nucleic Acids 12:568-577

20. Winbanks CE, Beyer C, Hagg A, Qian H, Sepulveda PV, Gregorevic P (2013) miR-206 represses hypertrophy of myogenic cells but not muscle fibers via inhibition of HDAC4. PLOS ONE 8(9):e73589

21. Huang QK, Qiao HY, Fu MH, Li G, Li WB, Chen Z, Wei J, Liang BS (2016) MiR-206 attenuates denervation-induced skeletal muscle atrophy in rats through regulation of satellite cell differentiation via TGF-beta1, Smad3, and HDAC4 signaling. Med Sci Monit 22:1161-1170

22. D'Souza RF, Markworth JF, Aasen KMM, Zeng N, Cameron-Smith D, Mitchell CJ (2017) Acute resistance exercise modulates microRNA expression profiles: combined tissue and circulatory targeted analyses. PLOS ONE 12(7):e0181594

23. Margolis LM, McClung HL, Murphy NE, Carrigan CT, Pasiakos SM (2017) Skeletal muscle myomiR are differentially expressed by endurance exercise mode and combined essential amino acid and carbohydrate supplementation. Front Physiol 8:182

24. McGee SL, Sparling D, Olson AL, Hargreaves M (2006) Exercise increases MEF2- and GEF DNA-binding activity in human skeletal muscle. FASEB J 20(2):348-349

25. Cohen TJ, Waddell DS, Barrientos T, Lu Z, Feng G, Cox GA, Bodine SC, Yao TP (2007) The histone deacetylase HDAC4 connects neural activity to muscle transcriptional reprogramming. J Biol Chem 282(46):33752-33759

26. Tang H, Macpherson P, Marvin M, Meadows E, Klein WH, Yang XJ, Goldman D (2009) A histone deacetylase 4/myogenin positive feedback loop coordinates denervation-dependent gene induction and suppression. Mol Biol Cell 20(4):1120-1131

\section{Publisher's Note}

Springer Nature remains neutral with regard to jurisdictional claims in published maps and institutional affiliations.
Ready to submit your research? Choose BMC and benefit from:

- fast, convenient online submission

- thorough peer review by experienced researchers in your field

- rapid publication on acceptance

- support for research data, including large and complex data types

- gold Open Access which fosters wider collaboration and increased citations

- maximum visibility for your research: over $100 \mathrm{M}$ website views per year

At $\mathrm{BMC}$, research is always in progress.

Learn more biomedcentral.com/submissions 